\title{
Screen pseudo slant lightlike submanifolds of golden semi-Riemannian manifolds
}

\author{
Bilal Eftal Acet (1D \\ Faculty of Arts and Sciences, Department of Mathematics, Adryaman University, Adryaman, Turkey
}

\begin{abstract}
In this article, we examine the term of screen pseudo-slant lightlike submanifolds of a golden semi-Riemannian manifold. Also, we obtain an example. We give some characterizations about the geometry of such submanifolds.
\end{abstract}

Mathematics Subject Classification (2010). 53C15, 53C25, 53C35

Keywords. semi-Riemannian manifold, golden ratio, lightlike submanifold

\section{Introduction}

From ancient times, the golden proportion has played very important role in architecture, arts, music etc. J. Kepler described golden structure which was revealed by the golden proportion. The number $\sigma=\frac{1+\sqrt{5}}{2}$ which is the real positive root of the equation

$$
x^{2}-x-1=0,
$$

is the golden proportion.

In [16], golden Riemannian manifolds were introduced by M. Crasmereanu and C.E. Hretcanu (see also $[17,18]$ ). In [2], the authors investigate the integrability of such manifolds. Also, the constancy of maps between golden Riemannian manifolds was introduced in [7]. On a golden Riemannian manifold, totally umbilical semi-invariant submanifolds were studied in [9]. Moreover, some types of lightlike submanifolds of metallic semiRiemannian manifolds were examined in [10].

A golden structure $P$ on a semi-Riemannian manifold $\bar{M}$ is defined as

$$
P^{2}-P-I=0,
$$

and if

$$
\bar{g}(P U, W)=\bar{g}(U, P W)
$$

then the semi-Riemannian metric is called $P$-compatible and $(\bar{M}, \bar{g}, P)$ is called a golden semi-Riemannian manifold [19].

In differential geometry, lightlike submanifolds of semi-Riemannian manifolds are an important research topic. This theory is developed by [12] (see also [15]). Then many authors studied lightlike submanifolds on different spaces $([4,5,13,14,21])$. Moreover, on golden semi-Riemannian manifolds, lightlike submanifolds have been reported by many mathematicians (see $[8,11,20]$ ).

Email address: eacet@adiyaman.edu.tr

Received: 10.07.2019; Accepted: 23.03.2020 
In [6], B.Y. Chen defined slant immersions in complex geometry. In [3], A. Lotta introduced the concept of slant immersions of a Riemannian manifold into an almost contact metric manifold. Also, in [1] bi-slant submanifolds with the notion of pseudo-slant submanifolds were introduced.

The purpose of this article is to study screen pseudo-slant lightlike submanifolds of a golden semi-Riemannian manifold. The article is arranged as follows. In Section 2 there are some basic definitions about lightlike submanifolds and golden semi-Riemannian manifolds. In Section 3, we give the definition of a screen pseudo-slant lightlike submanifold and obtain a non-trivial example. In the last section, we obtain main results of our paper.

\section{Preliminaries}

A submanifold $\left(M^{m}, g\right)$ immersed in a semi-Riemannian manifold $\left(\bar{M}^{m+n}, \bar{g}\right)$ is called a lightlike submanifold [12], if the metric $g$ induced from $\bar{g}$ is degenerate and the radical distribution $\operatorname{RadTM}$ is of rank $r, 1 \leq r \leq m$. Assume that $S(T M)$ is a screen distribution which is a semi-Riemannian orthogonal complementary distribution of $\operatorname{RadTM}$, that is,

$$
T M=S(T M) \perp R a d T M .
$$

Considering a screen transversal vector bundle $S\left(T M^{\perp}\right)$, which is a semi-Riemannian complementary vector bundle of $\operatorname{RadTM}$ in $T M^{\perp}$. For every local basis $\left\{E_{i}\right\}$ of $\operatorname{RadTM}$, there exists a local null frame $\left\{N_{i}\right\}$ of sections with values in the orthogonal complement of $S\left(T M^{\perp}\right)$ in $\left(S\left(T M^{\perp}\right)\right)^{\perp}$ such that

$$
\bar{g}\left(N_{i}, E_{i}\right)=\delta_{i j} \quad \text { and } \quad \bar{g}\left(N_{i}, N_{j}\right)=0,
$$

it follows that there exists a lightlike transversal vector bundle $\operatorname{lt}(T M)$ locally spanned by $\left\{N_{i}\right\}[12]$.

Assume that $\operatorname{tr}(T M)$ is a complementary (but not orthogonal) vector bundle to $T M$ in $\left.T \bar{M}\right|_{M}$. Then, we get

$$
\begin{gathered}
\operatorname{tr}(T M)=\operatorname{lt} T M \perp S\left(T M^{\perp}\right), \\
\left.T \bar{M}\right|_{M}=T M \oplus \operatorname{tr}(T M),
\end{gathered}
$$

which gives

$$
T \bar{M}=S(T M) \perp\{\operatorname{Rad} T M \oplus \operatorname{ltr}(T M)\} \perp S\left(T M^{\perp}\right) .
$$

The Gauss and Weingarten formulae are given as

$$
\begin{aligned}
& \bar{\nabla}_{U} V=\nabla_{U} V+h(U, V), \\
& \bar{\nabla}_{U} N=-A_{N} U+\nabla_{U}^{t} N,
\end{aligned}
$$

for all $U, V \in \Gamma(T M)$ and $N \in \Gamma(\operatorname{ltr}(T M))$, where $\nabla_{U} V, A_{N} U \in \Gamma(T M)$ and $h(U, V)$, $\nabla_{U}^{\frac{\perp}{U}} N \in \Gamma(\operatorname{tr}(T M)) . \bar{\nabla}, \nabla$, and $\nabla^{t}$ are linear connections on $T \bar{M}, T M$, and $\operatorname{tr}(T M)$, respectively.

In view of (2.5) and (2.6), for all $U, V \in \Gamma(T M), N \in \Gamma(\operatorname{ltr}(T M))$, and $W \in \Gamma\left(S\left(T M^{\perp}\right)\right)$, we get

$$
\begin{aligned}
\bar{\nabla}_{U} V & =\nabla_{U} V+h^{l}(U, V)+h^{s}(U, V), \\
\bar{\nabla}_{U} N & =-A_{N} U+\nabla_{U}^{l} N+D^{s}(U, N), \\
\bar{\nabla}_{U} W & =-A_{W} U+\nabla_{U}^{s} W+D^{l}(U, W),
\end{aligned}
$$

where

$$
\begin{array}{cc}
h^{l}(U, V)=L\left(h^{l}(U, V)\right), & h^{s}(U, V)=S\left(h^{s}(U, V)\right), \\
D^{l}(U, W)=L\left(\nabla_{U}^{t} W\right), & D^{s}(U, N)=S\left(\nabla_{U}^{t} N\right),
\end{array}
$$

where $L$ and $S$ are the projection morphism of $\operatorname{tr}(T M)$ on $\operatorname{ltr}(T M)$ and $S\left(T M^{\perp}\right)$, respectively. $\nabla^{l}$ and $\nabla^{s}$ are linear connections on $\operatorname{ltr}(T M)$ and $S\left(T M^{\perp}\right)$ called the lightlike connection and screen transversal connection on $M$, respectively. 
By the use of $(2.5),(2.7) \sim(2.9)$, and metric connection $\bar{\nabla}$, we arrive at

$$
\begin{gathered}
\bar{g}\left(h^{s}(U, V), W\right)+\bar{g}\left(V, D^{l}(U, W)\right)=\bar{g}\left(A_{W} U, V\right), \\
\bar{g}\left(D^{s}(U, N), W\right)=\bar{g}\left(N, A_{W} U\right) .
\end{gathered}
$$

We will denote the projection of $T M$ on $S(T M)$ by $P$. For any $U, V \in \Gamma(T M)$ and $E \in \Gamma(\operatorname{RadTM})$, we get

$$
\begin{gathered}
\nabla_{U} P V=\nabla_{U}^{*} P V+h^{*}(U, P V), \\
\nabla_{U} E=-A_{E}^{*} U+\nabla_{U}^{* t} E .
\end{gathered}
$$

From (2.12) with (2.13), we have

$$
\begin{gathered}
\bar{g}\left(h^{l}(U, P V), E\right)=g\left(A_{E}^{*} U, P V\right), \\
\bar{g}\left(h^{*}(U, P V), N\right)=g\left(A_{N} U, P V\right), \\
\bar{g}\left(h^{l}(U, E), E\right)=0, \quad A_{E}^{*} E=0 .
\end{gathered}
$$

By using (2.7), we find

$$
\left.\left(\nabla_{U} \bar{g}\right)(V, Z)=\bar{g}\left(h^{l}(U, V), Z\right)+\bar{g}^{l}(U, Z), V\right) .
$$

So, $\nabla$ is not a metric connection.

Let $(\bar{M}, \bar{g})$ be a semi-Riemannian manifold and $P$ be golden structure on $\bar{M}$. If

$$
\bar{g}(P U, V)=\bar{g}(U, P V),
$$

holds, then $(\bar{M}, \bar{g}, P)$ called a golden semi-Riemannian manifold. Equation (2.18) is equivalent to

$$
\bar{g}(P U, P V)=\bar{g}(P U, V)+\bar{g}(U, V) .
$$

Throughout this article, we suppose that $\bar{\nabla} P=0$.

\section{Screen pseudo-slant lightlike submanifolds}

Definition 3.1. Let $M$ be a lightlike submanifold of a golden semi-Riemannian manifold $\bar{M}$. If the following conditions are satisfied then $M$ is called a screen pseudo-slant submanifold of a golden semi-Riemannian manifold $\bar{M}$.

i) The radical distribution $\operatorname{RadTM}$ is an invariant distribution with respect to $P$, i.e.,

$$
P(\operatorname{RadTM})=\operatorname{RadTM} .
$$

ii) There exist $\stackrel{\circ}{D}$ and $\hat{D}$ non-degenerate orthogonal distributions on $M$ such that

$$
S(T M)=\stackrel{\circ}{D} \perp \hat{D} .
$$

iii) The distribution $\stackrel{\circ}{D}$ is anti-invariant, i.e.,

$$
P(\stackrel{\circ}{D}) \subset S\left(T M^{\perp}\right) \text {. }
$$

$i v$ ) The distribution $\hat{D}$ is slant with angle $\theta\left(\neq \frac{\pi}{2}\right)$, i.e., for each $x \in M$ and each non-zero vector $X \in(\hat{D})_{x}$, the angle $\theta$ between $P X$ and the vector subspace $(\hat{D})_{x}$ is a constant $\left(\neq \frac{\pi}{2}\right)$, which is independent of the choice of $x \in M$ and $X \in(\hat{D})_{x}$.

This constant angle $\theta$ is called the slant angle of distribution $\hat{D}$. A screen pseudo-slant lightlike submanifold is said to be proper if $\stackrel{\circ}{D} \neq\{0\}, \hat{D} \neq\{0\}$ and $\theta \neq 0$.

In view of above definition, we arrive at

$$
T M=\operatorname{Rad} T M \perp \stackrel{D}{\perp} \hat{D} .
$$


Example 3.2. Let $\left(\mathbb{R}_{5}^{10}, \bar{g}\right)$ be a semi-Riemannian manifold with signature $(-, \ldots,-,+, \ldots,+)$ and $\left(x_{1}, x_{2}, \ldots, x_{10}\right)$ be standard coordinate system of $\mathbb{R}_{5}^{10}$.

Taking

$$
P\left(x_{1}, \ldots, x_{10}\right)=\left(\begin{array}{c}
\sigma x_{1},(1-\sigma) x_{2},(1-\sigma) x_{3},(1-\sigma) x_{4},(1-\sigma) x_{5} \\
(1-\sigma) x_{6}, \sigma x_{7},(1-\sigma) x_{8},(1-\sigma) x_{9},(1-\sigma) x_{10}
\end{array}\right)
$$

then $P$ is a golden structure on $\mathbb{R}_{5}^{10}$.

Assume that $M$ is a submanifold of $\mathbb{R}_{5}^{10}$ given by

$$
\begin{gathered}
x_{1}=x_{7}=\omega^{1}, \quad x_{2}=\sigma \cos \alpha \omega^{5}+\cos \alpha \omega^{6}, \quad x_{3}=\sigma \omega^{2}, \\
x_{4}=\sigma \omega^{3}, \quad x_{5}=\sigma \omega^{4}, \quad x_{6}=\sigma \sin \alpha \omega^{5}+\sin \alpha \omega^{6}, \\
x_{8}=(1-\sigma) \omega^{2}, \quad x_{9}=(1-\sigma) \omega^{3}, \quad x_{10}=(1-\sigma) \omega^{4} .
\end{gathered}
$$

Then $T M=S p\left\{Z_{1}, Z_{2}, Z_{3}, Z_{4}, Z_{5}, Z_{6}\right\}$, where

$$
\begin{gathered}
Z_{1}=\frac{\partial}{\partial x_{1}}+\frac{\partial}{\partial x_{7}}, \quad Z_{2}=\sigma \frac{\partial}{\partial x_{3}}+(1-\sigma) \frac{\partial}{\partial x_{8}}, \\
Z_{3}=\sigma \frac{\partial}{\partial x_{4}}+(1-\sigma) \frac{\partial}{\partial x_{9}}, \quad Z_{4}=\sigma \frac{\partial}{\partial x_{5}}+(1-\sigma) \frac{\partial}{\partial x_{10}}, \\
Z_{5}=\sigma \cos \alpha \frac{\partial}{\partial x_{2}}+\sigma \sin \alpha \frac{\partial}{\partial x_{6}}, \quad Z_{6}=\cos \alpha \frac{\partial}{\partial x_{2}}+\sin \alpha \frac{\partial}{\partial x_{6}} .
\end{gathered}
$$

Thus, $\operatorname{RadTM}=\operatorname{Sp}\left\{Z_{1}\right\}$ and $S(T M)=S p\left\{Z_{2}, Z_{3}, Z_{4}, Z_{5}, Z_{6}\right\}$ and $\operatorname{ltr}(T M)$ is spanned by

$S\left(T M^{\perp}\right)$ is spanned by

$$
N=-\frac{1}{2}\left(\frac{\partial}{\partial x_{1}}-\frac{\partial}{\partial x_{7}}\right) .
$$

$$
\begin{gathered}
W_{1}=-\frac{\partial}{\partial x_{3}}+(1-\sigma)^{2} \frac{\partial}{\partial x_{8}}, \quad W_{2}=-\frac{\partial}{\partial x_{4}}+(1-\sigma)^{2} \frac{\partial}{\partial x_{9}}, \\
W_{3}=-\frac{\partial}{\partial x_{5}}+(1-\sigma)^{2} \frac{\partial}{\partial x_{10}} .
\end{gathered}
$$

It follows that $P Z_{1}=\sigma Z_{1}$, which implies that $\operatorname{RadTM}$ is invariant. Also, we can state that $\stackrel{\circ}{D}=\left\{Z_{2}, Z_{3}, Z_{4}\right\}$ such that $P Z_{2}=W_{1}, P Z_{3}=W_{2}, P Z_{4}=W_{3}$, which gives $\stackrel{\circ}{D}$ is anti-invariant and $\hat{D}=\left\{Z_{5}, Z_{6}\right\}$ is a slant distribution with slant angle $2 \alpha$. Therefore $M$ is a screen pseudo-slant lightlike submanifold of $\mathbb{R}_{5}^{10}$.

For any vector field $U \in \Gamma(T M)$ tangent to $M$, we take

$$
P U=R U+T U \text {, }
$$

where $R U$ and $T U$ are the tangential and the transversal part of $P U$, respectively. We denote the projections on $\operatorname{RadTM}, \stackrel{\circ}{D}$ and $\hat{D}$ in $T M$ by $R_{1}, R_{2}$, and $R_{3}$, respectively. Similarly, we show that the projections of $\operatorname{tr}(T M)$ on $\operatorname{lt}(T M), P(\stackrel{\circ}{D})$ and $D^{\prime}$ by $Q_{1}, Q_{2}$, and $Q_{3}$, respectively, where $D^{\prime}$ is a non-degenerate orthogonal complementary subbundle of $P(\stackrel{\circ}{D})$ in $S\left(T M^{\perp}\right)$. So, for any $U \in \Gamma(T M)$, we have

$$
U=R_{1} U+R_{2} U+R_{3} U .
$$

Applying $P$ to (3.3), we get

$$
P U=P R_{1} U+P R_{2} U+P R_{3} U
$$

which yields

$$
P U=P R_{1} U+P R_{2} U+w R_{3} U+T R_{3} U,
$$

where $w R_{3} U$ and $T R_{3} U$ denotes the tangential and the transversal component of $P R_{3} U$. So, we arrive at

$$
\begin{array}{ll}
P R_{1} U \in \Gamma(R a d T M), & P R_{2} U \in \Gamma(P(\stackrel{\circ}{D})) \subset S\left(T M^{\perp}\right), \\
w R_{3} U \in \Gamma(\hat{D}), & T R_{3} U \in \Gamma\left(D^{\prime}\right) .
\end{array}
$$

Also, for any $W \in \Gamma(\operatorname{tr}(T M))$, we get

$$
W=f_{1} W+f_{2} W+f_{3} W .
$$


Applying $P$ to (3.5), we have

$$
P W=P f_{1} W+P f_{2} W+P f_{3} W,
$$

which yields

$$
P W=P f_{1} W+P f_{2} W+B f_{3} W+C f_{3} W,
$$

where $B f_{3} W$ and $C f_{3} W$ denotes the tangential and the transversal component of $P f_{3} W$. Thus we get

$$
\begin{array}{ll}
P f_{1} W \in \Gamma(\operatorname{ltr}(T M)), & P f_{2} W \in \Gamma(\hat{D}), \\
B f_{3} W \in \Gamma(\hat{D}), & C f_{3} W \in \Gamma\left(D^{\prime}\right) .
\end{array}
$$

Now, using (3.4) and (3.6) with (2.7) (2.9), we obtain following;

$$
\begin{aligned}
& \nabla_{U}^{* t} P R_{1} V+R_{1}\left(\nabla_{U} w R_{3} V\right)=R_{1}\left(A_{T R_{3} V} U\right)+R_{1}\left(A_{P R_{2} V} U\right) \\
& +P R_{1} \nabla_{U} V \text {, } \\
& R_{2}\left(A_{P R_{1} V}^{*} U\right)+R_{2}\left(A_{P R_{2} V}^{*} U\right)+R_{2}\left(A_{T R_{3} V}^{*} U\right)=R_{2}\left(\nabla_{U} w R_{3} V\right) \\
& -P f_{1} h^{s}(U, V) \text {, } \\
& R_{3}\left(A_{P R_{1} V}^{*} U\right)+R_{3}\left(A_{P R_{2} V}^{*} U\right)+R_{3}\left(A_{T R_{3} V}^{*} U\right)=R_{3}\left(\nabla_{U} w R_{3} V\right) \\
& -w R_{3}\left(\nabla_{U} V\right)-B f_{3} h^{s}(U, V) \\
& h^{l}\left(U, P R_{1} V\right)+D^{l}\left(U, P R_{2} V\right)+h^{l}\left(U, w R_{3} V\right)+D^{l}\left(U, T R_{3} V\right)=P h^{l}(U, V) \text {, } \\
& f_{2} \nabla_{U}^{s} P R_{2} V+f_{2} \nabla_{U}^{s} T R_{3} V=P R_{2} \nabla_{U} V-f_{2} h^{s}\left(U, P R_{1} V\right) \\
& -f_{2} h^{s}\left(U, w R_{3} V\right) \text {, } \\
& f_{3} \nabla_{U}^{s} P R_{2} V+f_{3} \nabla_{U}^{s} T R_{3} V-T R_{3} \nabla_{U} V=C f_{3} h^{s}(U, V)-f_{3} h^{s}\left(U, w R_{3} V\right) \\
& -f_{3} h^{s}\left(U, P R_{1} V\right) \text {. }
\end{aligned}
$$

\section{Main theorems}

In this section, we give the main results of our article.

Theorem 4.1. Let $M$ be a screen pseudo-slant lightlike submanifold of a golden semiRiemannian manifold $\bar{M}$. Then the distribution RadTM is integrable if and only if, for all $E_{1}, E_{2} \in \Gamma(\operatorname{RadTM})$,

i) $f_{2} h^{s}\left(E_{2}, P R_{1} E_{1}\right)=f_{2} h^{s}\left(E_{1}, P R_{1} E_{2}\right)$,

ii) $f_{3} h^{s}\left(E_{2}, P R_{1} E_{1}\right)=f_{3} h^{s}\left(E_{1}, P R_{1} E_{2}\right)$,

iii) $R_{3} A_{P R_{1} E_{1}}^{*} E_{2}=R_{3} A_{P R_{1} E_{2}}^{*} E_{1}$.

Proof. Assume that $M$ is a screen pseudo-slant lightlike submanifold of a golden semiRiemannian manifold $\bar{M}$ and $E_{1}, E_{2} \in \Gamma(\operatorname{Rad} T M)$.

i) In view of (3.11), we get

$$
P R_{2} \nabla_{E_{1}} E_{2}=f_{2} h^{s}\left(E_{1}, P R_{1} E_{2}\right) .
$$

Interchanging $E_{1}$ to $E_{2}$, we obtain

$$
P R_{2} \nabla_{E_{2}} E_{1}=f_{2} h^{s}\left(E_{2}, P R_{1} E_{1}\right) .
$$

From (4.1) and (4.2), we obtain

$$
P R_{2}\left[E_{1}, E_{2}\right]=f_{2}\left(h^{s}\left(E_{1}, P R_{1} E_{2}\right)-h^{s}\left(E_{2}, P R_{1} E_{1}\right)\right) \text {. }
$$

ii) Similarly, by the use of (3.12), we have

$$
f_{3} h^{s}\left(E_{1}, P R_{1} E_{2}\right)=C f_{3} h^{s}\left(E_{1}, E_{2}\right)+T R_{3} \nabla_{E_{1}} E_{2},
$$

from which we get

$$
f_{3} h^{s}\left(E_{2}, P R_{1} E_{1}\right)=C f_{3} h^{s}\left(E_{2}, E_{1}\right)+T R_{3} \nabla_{E_{2}} E_{1} .
$$


From (4.3) and (4.4), we find

$$
T R_{3}\left[E_{1}, E_{2}\right]=f_{3}\left(h^{s}\left(E_{1}, P R_{1} E_{2}\right)-h^{s}\left(E_{2}, P R_{1} E_{1}\right)\right) .
$$

iii) Moreover, from (3.9), we have

$$
R_{3}\left(A_{P R_{1} E_{1}}^{*} E_{2}\right)=-w R_{3} \nabla_{E_{1}} E_{2}-B f_{3} h^{s}\left(E_{1}, E_{2}\right),
$$

from which, we get

$$
R_{3} A_{P R_{1} E_{2}}^{*} E_{1}=-w R_{3} \nabla_{E_{2}} E_{1}-B f_{3} h^{s}\left(E_{2}, E_{1}\right) .
$$

From (4.5) and (4.6), we obtain

$$
w R_{3}\left[E_{1}, E_{2}\right]=R_{3}\left(A_{P R_{1} E_{1}}^{*} E_{2}\right)-R_{3}\left(A_{P R_{1} E_{2}}^{*} E_{1}\right) .
$$

So, we arrive at the required equations.

Theorem 4.2. Let $M$ be a screen pseudo-slant lightlike submanifold of a golden semiRiemannian manifold $\bar{M}$. Then the distribution $\stackrel{D}{D}$ is integrable if and only if, for all $U, V \in \Gamma(\stackrel{D}{)})$

i) $R_{1}\left(A_{P R_{2} U} V\right)=R_{1}\left(A_{P R_{2} V} U\right)$ and $R_{3}\left(A_{P R_{2} U} V\right)=R_{3}\left(A_{P R_{2} V} U\right)$,

ii) $f_{3}\left(\nabla_{V}^{s} P R_{2} U\right)=f_{3}\left(\nabla_{U}^{s} P R_{2} V\right)$.

Proof. Assume that $M$ is a screen pseudo-slant lightlike submanifold of a golden semiRiemannian manifold $\bar{M}$ and $U, V \in \Gamma(\stackrel{D}{)})$.

i) By the use of (3.7), we get

$$
R_{1}\left(A_{P R_{2} U} V\right)=-P R_{1} \nabla_{U} V .
$$

Interchanging $U$ and $V$, we have

$$
R_{1}\left(A_{P R_{2} V} U\right)=-P R_{1} \nabla_{V} U .
$$

From (4.1) and (4.2), we obtain

$$
R_{1}\left(A_{P R_{2} U} V\right)-R_{1}\left(A_{P R_{2} V} U\right)=P R_{1}[U, V] .
$$

In view of $(3.9)$, we get

$$
R_{3}\left(A_{P R_{2} U} V\right)+B f_{3} h^{s}(U, V)=-w R_{3}\left(\nabla_{U} V\right),
$$

from which we have

$$
R_{3}\left(A_{P R_{2} U} V\right)-R_{3}\left(A_{P R_{2} V} U\right)=w R_{3}[U, V] .
$$

ii) Moreover, using (3.12), we have

$$
f_{3} \nabla_{U}^{s} P R_{2} V+C f_{3} h^{s}(U, V)=T R_{3} \nabla_{U} V,
$$

from which, we arrive at

$$
f_{3} \nabla_{U}^{s} P R_{2} V-f_{3} \nabla_{V}^{s} P R_{2} U=T R_{3}[U, V] .
$$

Thus, we obtain the desired results.

Theorem 4.3. Let $M$ be a screen pseudo-slant lightlike submanifold of a golden semiRiemannian manifold $\bar{M}$. Then the distribution $\hat{D}$ is integrable if and only if, for all $U, V \in \Gamma(\hat{D})$,

i) $R_{1}\left(\nabla_{U} w R_{3} V-\nabla_{V} w R_{3} U\right)=R_{1}\left(A_{T R_{3} V} U-A_{T R_{3} U} V\right)$,

ii) $f_{2}\left(\nabla_{U}^{s} T R_{3} V-\nabla_{V}^{s} T R_{3} U\right)=f_{2}\left(h^{s}\left(V, w R_{3} U\right)-h^{s}\left(U, w R_{3} V\right)\right)$. 
Proof. Assume that $M$ is a screen pseudo-slant lightlike submanifold of a golden semiRiemannian manifold $\bar{M}$ and $U, V \in \Gamma(\hat{D})$.

i) If we consider (3.7), we have

$$
R_{1}\left(\nabla_{U} w R_{3} V\right)=R_{1}\left(A_{T R_{3} V} U\right)+P R_{1} \nabla_{U} V .
$$

Interchanging $U$ and $V$, we have

$$
R_{1}\left(\nabla_{V} w R_{3} U\right)=R_{1}\left(A_{T R_{3} U} V\right)+P R_{1} \nabla_{V} U .
$$

In view of (4.9) with (4.10), we find

$$
R_{1}\left(\nabla_{U} w R_{3} V-\nabla_{V} w R_{3} U\right)-R_{1}\left(A_{T R_{3} V} U-A_{T R_{3} U} V\right)=P R_{1}[U, V] .
$$

ii) Also, using (3.11), we get

$$
f_{2} \nabla_{U}^{s} T R_{3} V+f_{2} h^{s}\left(U, w R_{3} V\right)=P R_{2} \nabla_{U} V
$$

with

$$
f_{2} \nabla_{V}^{s} T R_{3} U+f_{2} h^{s}\left(V, w R_{3} U\right)=P R_{2} \nabla_{V} U .
$$

From last two equations, we arrive at

$$
f_{2}\left(\nabla_{U}^{s} T R_{3} V-\nabla_{V}^{s} T R_{3} U\right)+f_{2}\left(h^{s}\left(U, w R_{3} V\right)-h^{s}\left(V, w R_{3} U\right)\right)=P R_{2}[U, V] .
$$

So, we obtain the required results.

Now, we find some conditions for foliations determined by distributions to be totally geodesic.

Theorem 4.4. Let $M$ be a screen pseudo-slant lightlike submanifold of a golden semiRiemannian manifold $\bar{M}$. Then the distribution RadTM defines a totally geodesic foliation if and only if

$$
\bar{g}\left(D^{l}\left(E_{1}, R_{2} Z\right)+D^{l}\left(E_{1}, T R_{3} Z\right), P E_{2}\right)=-\bar{g}\left(h^{l}\left(E_{1}, w R_{3} Z\right), P E_{2}\right),
$$

for all $E_{1}, E_{2} \in \Gamma(\operatorname{RadT} M)$ and $Z \in \Gamma(S(T M))$.

Proof. Let $M$ be a screen pseudo-slant lightlike submanifold of a golden semi-Riemannian manifold $\bar{M}$. We know that $\operatorname{RadTM}$ defines a totally geodesic foliation if and only if

$$
\nabla_{E_{1}} E_{2} \in \Gamma(\operatorname{RadTM}),
$$

for all $E_{1}, E_{2} \in \Gamma(\operatorname{RadTM})$.

Because of $\bar{\nabla}$ is a metric connection, by the use of (2.7), (2.19) with (3.4), we have

$$
\bar{g}\left(\nabla_{E_{1}} E_{2}, Z\right)=\bar{g}\left(P \bar{\nabla}_{E_{1}} E_{2}, P Z\right)-\bar{g}\left(P \bar{\nabla}_{E_{1}} E_{2}, Z\right) .
$$

So, we get

$$
\begin{aligned}
\bar{g}\left(\nabla_{E_{1}} E_{2}, Z\right)= & \bar{g}\left(\bar{\nabla}_{E_{1}} P E_{2}, P Z\right)-\bar{g}\left(\bar{\nabla}_{E_{1}} P E_{2}, Z\right) \\
= & \bar{g}\left(\bar{\nabla}_{E_{1}} P R_{2} Z, P E_{2}\right)+\bar{g}\left(\bar{\nabla}_{E_{1}} w R_{3} Z, P E_{2}\right) \\
& +\bar{g}\left(\bar{\nabla}_{E_{1}} T R_{3} Z, P E_{2}\right)-\bar{g}\left(\bar{\nabla}_{E_{1}} P R_{2} Z, E_{2}\right) \\
& -\bar{g}\left(\bar{\nabla}_{E_{1}} w R_{3} Z, P E_{2}\right)-\bar{g}\left(\bar{\nabla}_{E_{1}} T R_{3} Z, E_{2}\right) \\
= & \bar{g}\left(D^{l}\left(E_{1}, P R_{2} Z\right), P E_{2}\right)+\bar{g}\left(h^{l}\left(E_{1}, w R_{3} Z\right), P E_{2}\right) \\
& +\bar{g}\left(D^{l}\left(E_{1}, T R_{3} Z\right), P E_{2}\right)-\bar{g}\left(D^{l}\left(E_{1}, P R_{2} Z\right), E_{2}\right) \\
& -\bar{g}\left(h^{l}\left(E_{1}, w R_{3} Z\right), E_{2}\right)-\bar{g}\left(D^{l}\left(E_{1}, T R_{3} Z\right), E_{2}\right) .
\end{aligned}
$$

In view of last equation, we get the proof of theorem.

Theorem 4.5. Let $M$ be a screen pseudo-slant lightlike submanifold of a golden semiRiemannian manifold $\bar{M}$. Then the distribution $\stackrel{D}{D}$ defines a totally geodesic foliation if and only if

i) $\bar{g}\left(h^{s}(U, w Z), P V\right)=-\bar{g}\left(\nabla_{U}^{s} T Z, P V\right)$, and $h^{s}(U, Z) \in \Gamma\left(D^{\prime}\right)$, 
ii) $D^{s}(U, P N)$ has no component in $P(\stackrel{\circ}{D})$ and $D^{s}(U, N) \in \Gamma\left(D^{\prime}\right)$, for all $U, V \in \Gamma(\stackrel{D}{)})$ and $Z \in \Gamma(\hat{D})$ and $N \in \Gamma(\operatorname{ltr}(T M))$.

Proof. Let $M$ be a screen pseudo-slant lightlike submanifold of a golden semi-Riemannian manifold $\bar{M}$. We know that $\stackrel{D}{D}$ defines a totally geodesic foliation if and only if

$$
\nabla_{U} V \in \Gamma(\stackrel{\circ}{D}),
$$

for all $U, V \in \Gamma(\stackrel{D}{)})$.

By the use of (2.7) with (2.19), we have

$$
\begin{aligned}
\bar{g}\left(\nabla_{U} V, Z\right)= & -\bar{g}\left(P \nabla_{U} Z, P V\right)+\bar{g}\left(\nabla_{U} Z, P V\right) \\
= & -\bar{g}\left(\bar{\nabla}_{U} P Z, P V\right)+\bar{g}\left(\bar{\nabla}_{U} Z, P V\right) \\
= & \bar{g}\left(h^{s}(U, w Z), P V\right)+\bar{g}\left(\nabla_{U}^{s} T Z, P V\right) \\
& +\bar{g}\left(h^{s}(U, Z), P V\right),
\end{aligned}
$$

which gives $(i)$.

Also, from (2.7) with (2.19), we get

$$
\begin{aligned}
\bar{g}\left(\nabla_{U} V, Z\right) & =-\bar{g}\left(\bar{\nabla}_{U} P N, P V\right)+\bar{g}\left(\bar{\nabla}_{U} N, P V\right) \\
& =-\bar{g}\left(D^{s}(U, P N), P V\right)+\bar{g}\left(D^{s}(U, N), P V\right),
\end{aligned}
$$

which implies $(i i)$.

Theorem 4.6. Let $M$ be a screen pseudo-slant lightlike submanifold of a golden semiRiemannian manifold $\bar{M}$. Then the distribution $\hat{D}$ defines a totally geodesic foliation if and only if

i) $A_{P Z} U$ has no component in $\hat{D}$ and $\nabla_{U}^{s} P Z \in \Gamma(\stackrel{D}{D})$,

ii) $A_{P N} U$ has no component in $\hat{D}$ and $D^{s}(U, P N) \in \Gamma(\stackrel{D}{D})$,

for all $U, V \in \Gamma(\hat{D})$ and $Z \in \Gamma(\stackrel{D}{)}$ and $N \in \Gamma(\operatorname{ltr}(T M))$.

Proof. Let $M$ be a screen pseudo-slant lightlike submanifold of a golden semi-Riemannian manifold $\bar{M}$. We know that $\hat{D}$ defines a totally geodesic foliation if and only if

$$
\nabla_{U} V \in \Gamma(\hat{D}),
$$

for all $U, V \in \Gamma(\hat{D})$.

In view of (2.7) with (2.19), we have

$$
\begin{aligned}
\bar{g}\left(\nabla_{U} V, Z\right)= & -\bar{g}\left(\bar{\nabla}_{U} P Z, P V\right)+\bar{g}\left(\bar{\nabla}_{U} P Z, V\right) \\
= & \bar{g}\left(A_{P Z} U, w V\right)+\bar{g}\left(\nabla_{U}^{s} P Z, T V\right) \\
& +\bar{g}\left(A_{P Z} U, V\right),
\end{aligned}
$$

which yields $(i)$.

On the other hand, using (2.7) with (2.19), we get

$$
\begin{aligned}
\bar{g}\left(\nabla_{U} V, Z\right)= & -\bar{g}\left(\bar{\nabla}_{U} P N, P V\right)+\bar{g}\left(\bar{\nabla}_{U} N, P V\right) \\
= & -\bar{g}\left(A_{P N} U, w V\right)+\bar{g}\left(D^{s}(U, P N), T V\right) \\
& -\bar{g}\left(A_{P N} U, V\right),
\end{aligned}
$$

which implies $(i i)$. 


\section{References}

[1] A. Carriazo, New Developments in Slant Submanifolds Theory, New Delhi, India, 2002.

[2] A. Gezer, N. Cengiz and A. Salimov, On integrability of golden Riemannian structures, Turk. J. Math. 37, 693-703, 2013.

[3] A. Lotta, Slant submanifolds in contact geometry, Bull. Math. Soc. Roumanie 39, 183-198, 1996.

[4] B.E. Acet, S. Yüksel Perktaş and E, Kılıç, On lightlike geometry of para-Sasakian manifolds, Sci. World J. Art. ID 696231, 2014.

[5] B.E. Acet, S. Yüksel Perktaş and E, Kılıç, Symmetric lightlike hypersurfaces of a para-Sasakian space form, An. Ştiint Al. I. Cuza Iaşi. Mat. (N.S.), 2, 915-926, 2016.

[6] B.Y. Chen, Geometry of Slant Submanifolds, Leuven, Katholike Univ. 1990.

[7] B. Şahin and M.A. Akyol, Golden maps between golden Riemannian manifolds and constancy of certain maps, Math Commun. 19, 333-342, 2014.

[8] F.E. Erdoğan, Screen transversal lightlike submanifolds of golden semi-Riemannian manifolds, arXiv:1805.06783v1.

[9] F.E. Erdoğan and C. Yıldırım, Semi-invariant submanifolds of golden Riemannian manifolds, AIP Conference Proceedings. 1833 (020044), doi:10.1063/1.4981692, 2017.

[10] F.E. Erdoğan, S. Yüksel Perktaş, B.E. Acet and A.M. Blaga, Screen transversal lightlike submanifolds of metallic semi-Riemannian manifolds, J. Geom. Phys. 142, 111120, 2019.

[11] F.E. Erdoğan, S. Yüksel Perktaş and B.E. Acet, Invariant lightlike submanifolds of golden semi-Riemannian manifolds, AIP Conference Proceedings. 1991 (020011), doi:10.1063/1.5047884, 2018.

[12] K.L. Duggal and A. Bejancu, Lightlike Submanifolds of Semi-Riemannian Manifolds and Applications, Mathematics and Its Applications, Kluwer Publisher, 1996.

[13] K.L. Duggal and B. Şahin, Generalized Cauchy-Riemann lightlike submanifolds of Kaehler manifolds, Acta Math. Hungar. 112, 107-130, 2006.

[14] K.L. Duggal and B. Şahin, Lightlike submanifolds of indefinite Sasakian manifolds, Int. J. Math. Math. Sci. Art. No. 57585, 2007.

[15] K.L. Duggal and B. Şahin, Differential Geometry of Lightlike Submanifolds, Frontiers in Mathematics, 2010.

[16] M. Crasmareanu and C.E. Hretcanu, Golden differential geometry, Chaos Solitons Fractals 38 (5), 1229-1238, 2008.

[17] M. Crasmareanu and C.E. Hretcanu, Applications of the golden ratio on Riemannian manifolds, Turk. J. Math. 33 (2), 179-191, 2009.

[18] M. Crasmareanu and C.E. Hretcanu, On the some invariant submanifolds in a Riemannian manifold with golden structure, An. Ştiint Al. I. Cuza Iaşi. Mat. (N.S.) 53 (1), 199-211, 2007.

[19] M. Özkan, Prolongations of golden structure to tangent bundles, Diff. Geo. Dynm. Syst. 16, 227-238, 2014.

[20] N. Önen Poyraz and E. Yaşar, Lightlike hypersurfaces of a golden semi-Riemannian manifold, Mediterr. J. Math. 14, Article number: 204, 2017.

[21] S. Yüksel Perktaş and F.E. Erdoğan, On generalized CR-lightlike submanifolds, Palestine J. Math. 8 (1), 200-208, 2019. 